\title{
Tangence
}

\section{L'écriture pour la jeunesse :de la production à la réception}

\section{Claire Le Brun et Monique Noël-Gaudreault}

Numéro 67, automne 2001

L'écriture pour la jeunesse : de la production à la réception

URI : https://id.erudit.org/iderudit/009611ar

DOI : https://doi.org/10.7202/009611ar

Aller au sommaire du numéro

Éditeur(s)

Presses de l'Université du Québec

ISSN

0226-9554 (imprimé)

1710-0305 (numérique)

Découvrir la revue

Citer cet article

Le Brun, C. \& Noël-Gaudreault, M. (2001). L'écriture pour la jeunesse :de la

production à la réception. Tangence, (67), 5-8. https://doi.org/10.7202/009611ar d'utilisation que vous pouvez consulter en ligne.

https://apropos.erudit.org/fr/usagers/politique-dutilisation/ 


\section{L'écriture pour la jeunesse : de la production à la réception \\ Claire Le Brun et Monique Noël-Gaudreault}

Dans l'histoire de la littérature pour la jeunesse, production littéraire définie par son public, la question des contraintes fixées par le lectorat s'est toujours posée avec acuité. Charles Perrault qui est, pour le domaine français, l'un des plus anciens témoins d'une conscience de la spécificité des cuvres pour la jeunesse, oppose, dans la préface des Contes de ma mère l'Oye (1697), les contes de l'Antiquité gréco-latine, destinés avant tout à plaire, et les "contes que nos aïeux ont inventés pour leurs enfants". Car ces derniers, explique-t-il, "ne les ont pas contés avec l'élégance et les agréments dont les Grecs et les Romains ont orné leurs Fables; mais ils ont toujours eu un très grand soin que leurs contes renfermassent une morale louable et instructive". Cette tension entre le plaisir esthétique et la formation morale, entre l'art et l'éthique, continue de marquer les débats sur la littérature pour la jeunesse.

La prise en compte des besoins propres aux différentes catégories de lecteurs se traduit, dès le XVIII ${ }^{e}$ siècle, par une division en groupes d'âges: que l'on songe au Magasin des enfants (1757) et au Magasin des adolescents (1760) de Madame Leprince de Beaumont. Les livres des garçons se distinguent aussi de ceux des filles. Il faudra attendre les années 1950 pour que les lectures évoluent peu à peu vers la mixité, suivant ainsi les nouvelles orientations pédagogiques.

Aujourd'hui, si les lectures d'enfance et de jeunesse sont officiellement "unisexes", elles se fragmentent en revanche en une complexité de catégories, variant souvent d'un éditeur à l'autre. Les publications pour la jeunesse se gagnent un public de plus en plus large en annexant de nouvelles tranches d'âge, depuis les "neuf mois à deux ans" jusqu'aux "jeunes adultes". Dans le même temps - retour du balancier? -, des expériences éditoriales visent à présenter le même produit aux enfants et aux adultes, qu'il s'agisse de l'album, du conte ou même du roman. La compartimentation du lectorat chez la majorité des éditeurs semble 
avoir pour pendant la porosité des frontières chez quelques autres.

Au Québec, La courte échelle, chef de file de l'édition pour la jeunesse, a franchi un pas décisif en 1996, en lançant la nouvelle collection " $16 / 96$ " pour les jeunes, et surtout moins jeunes, adultes, dont les premiers titres étaient signés par des auteurs bien connus en littérature jeunesse et souvent identifiés à cette maison. Un an plus tard, Québec/Amérique allait plus loin en lançant le roman préhistorique de Dominique Demers, Maina (1997), simultanément dans une collection pour adolescents et dans une collection pour adultes.

Ces tendances opposées qui coexistent au sein de l'édition définition de plus en plus précise du public visé et cahier de charges contraignant, d'une part; ouverture à un double lectorat, d'autre part - ne peuvent manquer d'influer sur les écrivains pour la jeunesse. Comment s'accommodent-ils de cet ensemble de règles explicites et implicites? de ce nouvel espace éditorial à la fois balisé et ouvert? Face à un public mouvant, quelle perception ont-ils d'eux-mêmes, de leur tâche, de leur place au sein de l'institution littéraire?

Des spécialistes de plusieurs disciplines (littérature, didactique, sociologie de la littérature) confrontent leurs réflexions sur l'écriture pour la jeunesse, en prenant comme fil conducteur la relation de l'auteur avec son ou ses publics: public choisi ou imposé, courtisé ou ignoré, qu'une décision éditoriale peut restreindre ou élargir, voire doubler.

Dans un vaste panorama d'œuvres marquantes des années 1990, Sandra L. Beckett montre que la perméabilité des frontières entre livre pour enfants et livre pour adultes est un phénomène commun à l'ensemble des pays occidentaux. En examinant les cas de J. K. Rowling et de Philip Pullmann au Royaume-Uni, de Le Clézio et de Tournier en France, de Martín Gaite en Espagne, pour n'en citer que quelques-uns, elle met en lumière les stratégies utilisées par les éditeurs et par les auteurs eux-mêmes, qui remanient le texte selon le public ou le destinent ouvertement à un double lectorat.

Quelle différence y a-t-il entre un "auteur jeunesse" et un écrivain? Le statut de celles et ceux qui écrivent à la fois pour les enfants et les adultes est-il différent du statut des auteurs qui se 
consacrent exclusivement à un lectorat juvênile? Édith Madore retrace les étapes de la constitution d'un champ littéraire de la jeunesse au Québec et examine les conséquences de cette autonomisation sur la légitimité des "auteurs jeunesse". Quelle perception ces derniers ont-ils d'eux-mêmes et de leur tâche? Se situant également dans la théorie bourdieusienne des champs, Jean-Denis Côté analyse les positions d'une trentaine d'acteurs du sous-champ de la littérature jeunesse (auteurs, éditeurs, professeurs, critiques, etc.) en se concentrant sur la question cruciale de la rémunération financière de l'œuvre littéraire. Flore Gervais, quant à elle, a donné la parole au lectorat cible. Une enquête effectuée auprès d'enfants de cinq à neuf ans dans un cadre scolaire fait apparaître une gamme de représentations mentales du livre et de l'écrit, qui vont en se complexifiant avec l'âge.

Quelles sont les modalités d'inscription du lecteur ou de la lectrice dans les textes destinés à la jeunesse? Trois articles abordent la question dans des perspectives complémentaires. En analysant l'incipit comme lieu stratégique de contact entre l'auteur et son public, dans un corpus de romans québécois de la première moitié du $\mathrm{xx}^{*}$ siècle, Johanne Prud'homme dégage un portrait du lecteur et de la lectrice visés au cours de cette période fondatrice. Revisitant le concept de Lecteur modèle proposé par Umberto Eco, concept opératoire particulièrement utile pour appréhender une production littéraire fortement déterminée par son destinataire, Noëlle Sorin observe que la pléthore de lectures mises à la disposition des enfants sont souvent de piètre qualité, les éditeurs recherchant avant tout, pour le lecteur empirique, la lisibilité. Selon l'auteure, une didactique de la lecture et de l'écriture littéraires devrait viser à réduire l'écart entre le Lecteur modèle institué par un texte de qualité et le lecteur empirique. Enfin, Lucie Guillemette examine un choix de romans québécois, parus entre le début des années 1960 et la fin des années 1990, et dont les héroïnes se définissent dans une pratique de la lecture et de l'écriture. L'article observe les procédés intertextuels et interdiscursifs mis en cuvre dans la construction du personnage autoreprésenté de l'adolescente et identifie les compétences requises du lectorat.

Les deux dernières études s'intéressent à des itinéraires d'auteurs qui réécrivent, adaptent, transposent pour des publics différents. Claire Le Brun observe le passage conjoint d'un genre littéraire à un autre et d'un public à un autre chez Jasmine Dubé, qui 
s'est essayée avec bonheur dans divers médias: théâtre, albums, télévision, romans. En se penchant sur les dialogues d'une série de récits destinés aux jeunes lecteurs, elle montre que l'écriture romanesque de Dubé, à l'instar de son théâtre, vise à ouvrir un dialogue entre l'enfant et l'adulte. Daniel Chouinard se penche sur la distinction entre roman pour la jeunesse et romans pour adultes qui tend à se complexifier dans les récentes publications de Dominique Demers, figure marquante du roman québécois pour la jeunesse; la publication de Maïna et de la série MarieTempête dans une collection jeunesse et dans une collection adultes a en effet effectué un véritable brouillage éditorial.

Qu'annoncent les prochaines décennies? Le livre expressément écrit pour enfants est-il voué à la disparition? La notion d'écrivain pour la jeunesse devient-elle anachronique? Il est encore difficile d'évaluer exactement la portée du crossover phenomenon décrit par Sandra L. Beckett. Laissons, pour le moment, le mot de la fin à Daniel Chouinard, qui souligne le bénéfice de ces transferts éditoriaux pour le lecteur adulte: celui-ci, "absous, rédimé", peut lire "l'excellent ouvrage pour adolescents [...] comme un vrai livre"! Plus que l'enfant ou l'adolescent, l'adulte - éditeur, parent, ou... auteur en quête de légitimation - ne serait-il pas le grand gagnant de la nouvelle donne éditoriale? 